\title{
Adaptive Intelligent Tutorial Dialogue in the BEETLE II System
}

\author{
Myroslava O. Dzikovska ${ }^{1}$, Amy Isard ${ }^{1}$, Peter Bell ${ }^{1}$, Johanna D. Moore ${ }^{1}$, \\ Natalie B. Steinhauser ${ }^{2}$, Gwendolyn E. Campbell ${ }^{2}$, \\ Leanne S. Taylor ${ }^{3}$, Simon Caine ${ }^{3}$, and Charlie Scott ${ }^{3, *}$ \\ ${ }^{1}$ School of Informatics, University of Edinburgh, Edinburgh, United Kingdom \\ $\{\mathrm{m}$.dzikovska, amy.isard, p.bell, j.moore $\}$ ded.ac.uk \\ ${ }^{2}$ Naval Air Warfare Center Training Systems Division, Orlando, FL, USA \\ ${ }^{3}$ Kaegan Corporation, 12000 Research Parkway, Orlando, FL 32826-2944
}

In this interactive event we present Beetle II, a tutorial dialogue system designed to accept unrestricted language input and to support experimentation with different approaches to tutoring. Encouraging students to produce explanations and giving them detailed feedback is important for effective learning (e.g., [2]). But adding this capability to existing ITS remains a major challenge, due to the limitations of the existing natural language processing techniques. Statistical approaches like Latent Semantic Analysis have been used to interpret long student explanations. However, they require extensive pre-authoring, including anticipating a range of possible correct and incorrect answers, and manually recording tutor's feedback for every possible tutoring situation.

The Beetle II tutor asks students to explain their reasoning and accepts complex sentence-long answers to such open-ended questions. It avoids extensive preauthoring by using a deep parser and interpreter, together with a tutoring and generation module, to automatically generate tutoring feedback adapted to the system's assessment of the student's answer and previous dialogue history.

The system has undergone a successful evaluation in 2009 [1], which found significant learning gains for students interacting with the system. We collected a rich data set which enables investigating various aspects of tutorial dialogue, e.g., differences between human-human and human-computer interaction; the impact of language understanding problems on learning gain and user satisfaction; ways to improve language understanding techniques and tutoring strategies for use in Intelligent Tutoring Systems. The event participants will interact with the system trying to complete an exercise and discover the correct answer.

\section{References}

1. Dzikovska, M., Bental, D., Moore, J.D., Steinhauser, N.B., Campbell, G.E., Farrow, E., Callaway, C.B.: Intelligent tutoring with natural language support in the Beetle II system. In: Proceedings of ECTEL 2010 (2010)

2. Nielsen, R.D., Ward, W., Martin, J.H.: Learning to assess low-level conceptual understanding. In: Proceedings 21st International FLAIRS Conference (2008)

* This work has been supported in part by US Office of Naval Research grants N000140810043 and N0001410WX20278. 